\title{
Synthetic Hydrogels Mimicking Basement Membrane Matrices to Promote Cell-Matrix Interactions
}

\author{
Ricardo Cruz-Acuña ${ }^{1,3}$ and Andrés J. García ${ }^{2,3}$
}

\begin{abstract}
Naturally-derived materials have been extensively used as 3D cellular matrices as their inherent bioactivity makes them suitable for the study of many cellular processes. Nevertheless, lot-to-lot variability, inability to decouple biochemical and biophysical properties and, in some types, their tumorderived nature limits their translational potential and reliability. One innovative approach to overcome these limitations has focused on incorporating bioactivity into cytocompatible, synthetic hydrogels that present tunable physicochemical properties. This review provides an overview of successful approaches to convey basement membrane-like bioactivity into 3D artificial hydrogel matrices in order to recapitulate cellular responses to native matrices. Recent advances involving biofunctionalization of synthetic hydrogels via incorporation of bioactive motifs that promote cell-matrix interactions and cell-directed matrix degradation will be discussed. This review highlights how the tunable physicochemical properties of biofunctionalized synthetic hydrogel matrices can be exploited to study the separate contributions of biochemical and biophysical matrix properties to different cellular processes.
\end{abstract}

\section{Introduction}

Cell-extracellular matrix (ECM) interactions transduce mechanical and biochemical signals that regulate diverse cellular processes during embryogenesis, tissue repair, homeostasis, and pathogenesis (Martins-Green and Bissell, 1995; Lukashev and Werb, 1998; Midwood et al., 2004; Streuli, 1999). Bissell, Mostov and others have pioneered the use of 3D collagen and laminin (i.e., Matrigel ${ }^{\mathrm{TM}}$ ) gels in organotypic cultures that recreate morphogenetic developmental programs observed in the organism (Barcellos-Hoff, 1989; O’Brien et al., 2001; Montesano et al., 1991). Similarly, an understanding of cellECM interactions has been a major goal for biomaterials science in order to engineer synthetic materials 
that can recapitulate complex ECM-mediated cellular responses. Although cytocompatible synthetic materials exhibit the advantage of tunable biophysical and biochemical properties, the adaptation of these materials as biologically active cellular matrices remains a challenge. Nevertheless, recent studies have demonstrated major advances in the development of engineered synthetic hydrogel matrices that can mimic ECM properties, specifically, but not limited to, basement membrane (BM) characteristics. These research advances address three fundamental questions of biomaterial science: (1) How can we integrate suitable biochemical cues into synthetic hydrogels that mimic cell-BM interactions? (2) Is it possible to recapitulate native cellular programs by tuning the physicochemical properties of synthetic hydrogel matrices? (3) What benefits do these engineered synthetic hydrogels provide over natural matrices?

\subsection{Basement Membrane}

The basement membrane (BM) is a specialized form of the ECM that is mainly composed of a mixture of glycoproteins (Kalluri, 2003). It is found adjacent to most epithelial and endothelial layers in the body as it serves as a structural support that separates cell monolayers from the underlying connective tissue. BM also dictates many cellular processes by specific interactions between BM components and cellular receptors (LeBleu et al., 2007).

The four major components of the BM are: type IV collagen, laminin, nidogen (formerly called entactin), and perlecan (Kalluri, 2003; LeBleu et al., 2007; Yurchenko, 2011). Each major component plays a different role in the structural assembly of the matrix as well as in its bioactivity (Yurchenko, 2011). The BM structure is characterized by self-assembly of type IV collagen and laminin into polymeric networks that provide structural stability. Type IV collagen is a non-fibrous collagen that forms a triple helix composed of three $\alpha$-chains, while laminin is the most abundant non-collagenous protein in the $\mathrm{BM}$ and its structure is characterized by a heterotrimeric three-pronged fork made of $\alpha-, \beta-$, and $\gamma-$ chains (LeBleu et al., 2007). At least twelve different laminin isoforms have been identified, resulting from the combination of five $\alpha$-, three $\beta$-, and three $\gamma$-chains (Siler et al., 2000; Engbring et al., 2003). Laminin isoforms provide different architectural variations to the BM that in turn create a functional diversity that contributes to BM tissue-specificity (Cheng et al., 1997; Kalluri, 2003). Moreover, laminin and type IV collagen contribute to matrix bioactivity by containing specific motifs that are primarily recognized by cell receptors such as integrins (Kalluri, 2003). Interactions between the $\alpha \beta$ integrin 
heterodimers and ECM peptide motifs mediate essential cellular processes such as cell migration, proliferation, and differentiation (Hynes, 2009; Huttenlocher and Horwitz, 2011; Hynes, 2014; Garcia, 2005) (Figure 1a). Furthermore, the type IV collagen and laminin networks are linked together by nidogen and perlecan, which are secreted by cells as single molecules and cannot self-assemble into a network (LeBleu et al., 2007) (Figure 1b).

\subsection{BM-like Natural 3D Matrices}

In an attempt to create materials that mimic the native basement membrane environment, matrices consisting of extracts or purified proteins (e.g. type I collagen) have been developed. These 3D natural matrices have been used for a wide range of studies to model epithelial cell morphogenesis (Yu et al., 2005) and migration (Wolf et al., 2009), cancer invasion (Gaggioli et al., 2007) and metastasis (NguyenNgoc et al., 2012), as well as many epithelial morphogenetic programs (Shamir and Ewald, 2014).

\subsubsection{MatrigelTM}

Matrigel $^{\mathrm{TM}}$ is the commercial name for a BM extract secreted by Engelbrecht-Holm-Swarm (EHS) mouse sarcoma cells. Matrigel ${ }^{\mathrm{TM}}$ is a complex mixture of over 1,000 proteins (Hughes et al., 2010). It primarily consists of type IV collagen, laminin and nidogen, which makes it the most BM-like natural matrix model (Hughes et al., 2010; Yurchenco, 2011). Epithelial morphogenesis (Peng et al., 2014) and oncogenesis (Debnath et al., 2003), as well as intestinal organoids generation (Sato and Clevers, 2013; Yui et al., 2012; Fatehullah et al., 2016) are current major areas that involve 3D cultures in Matrigel ${ }^{\mathrm{TM}}$. A study by Sato et al (2009) exemplifies the potential of Matrigel ${ }^{\mathrm{TM}}$ by establishing a 3D culture condition that generates intestinal crypt organoids from single intestinal stem cells in the absence of a cellular niche. After 4 days of encapsulation in Matrigel ${ }^{\mathrm{TM}}$, and supplemented with soluble proteins essential for crypt proliferation (R-spondin 1 and epidermal growth factor) and expansion (Noggin), the resulting multi-cellular structures comprise approximately 100 cells, which is consistent with the 12-hour cell cycle of proliferative crypt cells. Additionally, the presence of stem cells at the crypt bottom as well as four major differentiated epithelial cell types are present, demonstrating the ability of Matrigel ${ }^{\mathrm{TM}}$ to support intestinal epithelial expansion reminiscent of normal gut (Fatehullah et al., 2016).

\subsubsection{Laminin}


Laminins are highly biologically active molecules that can act as strong adhesive substrates for many cell types, and have been found to promote cell adhesion, migration, protease activity, proliferation, tumor growth, angiogenesis, and metastasis (Siler et al., 2000; Engbring et al., 2003; Colognato and Yurchenco, 2000). Among the more of than a dozen laminin isoforms, laminin-111 (also known as laminin-1; composed of $\alpha 1$-, $\beta 1$-, and $\gamma 1$-chains) is the most widely used for 2D and 3D culture systems as it can be isolated from EHS mouse tumors and it is commercially available (Siler et al., 2000; Kikkawa et al., 2013). The use of laminin-111 as a culture substrate has helped identify specific peptide motifs that promote malignant phenotypes by increasing tumor adhesion and migration, as well as tumor cell metastasis through induction of protease production (Engbring et al., 2003; Kikkawa et al., 2013; Turpeenniemi-Hujanen et al., 1985). Although laminin-111 is the most prominent and studied form of laminin, studies have provided insights into the importance of other laminin isoforms in the morphogenesis of different epithelial organs (Ekblom et al., 1998; Li et al., 2015; Durbeej et al., 1996).

\subsubsection{Other Naturally-derived 3D Matrices}

Other naturally derived materials that have been used as cellular matrices include fibrin, alginate and hyaluronic acid gels. Fibrin gels, which are products of the polymerization of fibrinogen by the proteolytic activity of thrombin, started as one of the first biomaterials used to prevent bleeding and promote wound healing (Janmey et al., 2016), and transitioned to a variety of biomedical applications including islet transplantation (Riopel et al., 2015). Alginate and hyaluronic acid gels, which are derived from marine brown algae and vertebrate connective tissues, respectively (Matricardi et al., 2013), are polysaccharides that have been extensively used as biomaterials for controlled release of drugs and tissue engineering applications (Lee and Mooney, 2012; Kim et al., 2011; Lam et al., 2014).

\subsection{Limitations of Natural Matrices}

Although 3D natural matrices have been found suitable for the study of many cellular processes, they are limited by lot-to-lot compositional and structural variability (Hughes et al., 2010) which decreases their reliability. Additionally, these matrices are limited by the inability to decouple mechanical and biochemical properties. For instance, a common approach to vary the mechanical properties is to change the bulk concentration (e.g., increase matrix density). However, these changes in bulk 
concentration inevitably alter other matrix properties, such as adhesive ligand density and fiber density/ structure. Furthermore, as Matrigel ${ }^{\mathrm{TM}}$ is a tumor-derived matrix, its clinical translational potential is limited for regenerative medicine applications. For these reasons, approaches have been pursued to overcome these limitations, including the combination of purified natural BM proteins with biocompatible synthetic hydrogels that exhibit defined mechanical properties (Beck et al., 2013; Hutson et al., 2011). However, although these materials have decoupled mechanical and biochemical properties, they still have limited translational potential. Therefore, as the field of biomaterials continues to advance, novel engineered synthetic hydrogel matrices that present independently-tunable BM-like bioactivity and mechanical properties have been developed, overcoming each of the aforementioned limitations of natural matrices.

\section{Engineered Synthetic Hydrogel Matrices}

Hydrogels are water-swollen crosslinked polymer networks that can be considered synthetic equivalents of ECM matrices. Commonly used hydrogel polymers for biomedical applications include poly(acrylic acid) (PAA), poly(ethylene glycol) (PEG), poly(vinyl alcohol) (PVA), polyacrylamide (PAAm), and polypeptides (Zhu, 2010). These mesh-like structures are held together by either covalent crosslinks or non-covalent interactions that include ionic interactions, hydrogen bonds, affinity and hydrophobic interactions, polymer crystallites, physical entanglements, or a combination of the above (Peppas et al., 2000).

\subsection{Physical Properties of Hydrogels}

Hydrogel stiffness and swelling behavior are two important physical characteristics that are inversely related and are primarily controlled by polymer density (Flory and Rehner, 1943; Anseth et al., 1995; Drury and Mooney, 2003). The swelling behavior of hydrogels involves a swelling force produced by the thermodynamic compatibility of the polymer chains and water, which is counterbalanced by the retractive forces exerted by its crosslinks (Flory and Rehner, 1943; Peppas et al., 2000). Swelling equilibrium is reached when these two forces are equal (Peppas et al., 2000). Consequently, a hydrogel with high (low) polymer density has a greater (smaller) number of crosslinks that cause increased (decreased) retractive forces that will result in less (more) swelling. Moreover, an opposite effect is followed in hydrogel stiffness, as polymer density is proportional to the matrix stiffness; a high polymer 
density would produce a denser (thus, stiffer) polymer network. Hydrogel elastic modulus is an important physical parameter to consider in the design of a synthetic cellular matrix as many studies demonstrate matrix stiffness-dependent cellular responses, such as extent of cell migration and differentiation (Lutolf et al., 2003; Das et al., 2015).

\subsection{Synthesis of Hydrogels}

Among several methods to produce synthetic hydrogel matrices, photo-polymerization and chemical reaction crosslinking stand out as two of the most widely used for biomedical applications (Hoffman, 2002; Zhu, 2010). Photo-polymerization involves the crosslinking of two water-soluble polymers (Fig. 2a), or copolymerization between one or more monomers and one multi-functional monomer (Fig. 2b), via photo-ionization of the monomers using light, often in the UV range. The high reactivity of the photo-ionized polymer components induces crosslinking reactions that produce a crosslinked network. As the polymerization is light-dependent, this technique grants in situ spatiotemporal control over the mechanical properties of the hydrogel and presentation of incorporated ligands (Zhu, 2010; Kloxin et al., 2010; Nguyen and West, 2010; Lee et al., 2014).

Chemical reaction crosslinking involves a direct chemical reaction between linear or branched polymer macromolecules (macromers) with a di-functional or multi-functional crosslinking agent. Each agent, which has a smaller molecular weight, crosslinks two or more macromers together forming a meshlike structured hydrogel (Hoffman, 2002) (Fig. 2c). Examples of chemical reactions used to produce hydrogels are chemical ligation (Hu et al., 2009), click chemistry (Polizzotti et al., 2008) and Michaeltype addition reaction (Phelps et al., 2012).

These two general hydrogel synthesis methods have been widely used for generating artificial 3D

cellular matrices as they allow easy incorporation of BM-like biochemical properties, which will be discussed in the next section. Although these synthesis techniques are based on covalently crosslinking the synthetic hydrogel constituents, there are other synthesis methods that involve crosslinking by noncovalent interactions that are common in naturally-derived hydrogels, such as alginate "ionotropic" hydrogels which are formed based on electrostatic interactions (Hoffman, 2002) (Figure 2d). 


\subsection{Incorporation of BM-like Properties}

The BM contains key biophysical and biochemical characteristics that are essential for epithelial and endothelial cell attachment and viability as well as numerous cellular processes that include proliferation, differentiation, and migration. These cellular processes are primarily mediated by cellmatrix interactions via integrins, the ability of cells to remodel their supporting matrix via degradation of their microenvironment, and by presentation of growth factors (Lu et al., 2011). Consequently, in order to incorporate bioactivity into artificial hydrogel matrices, successful approaches have focused on incorporating bioactive peptides derived from natural ECM. Three common themes explored in biofunctional hydrogels are (1) cell adhesive peptides, (2) protease-degradable crosslinkers, and (3) growth factor-binding domains in order to mimic native cell-BM interactions (Fig. 2e).

\subsubsection{Cell Adhesive Peptides}

Integrin binding to short peptide sequences present in BM proteins such as collagen and laminin provides cellular attachment and triggers signals that direct cell function, cell-cycle progression and expression of differentiated phenotypes (Hynes, 2002; Giancotti and Ruoslahti, 1999; Danen and Sonnenberg, 2003; Danen, 2005). Therefore, incorporating biofunctional short peptide sequences into 3D artificial hydrogel systems has been an effective method to promote integrin-mediated cellular functions. These peptide motifs have the advantage of being relatively stable, and they can be covalently tethered to the hydrogel polymer backbone at tunable densities via integration of reactive groups, yielding a biofunctionalized hydrogel (Zhu, 2010). The most widely used cell-adhesive motif is the short peptide arginine-glycine-aspartic acid (RGD); this oligopeptide was originally identified in fibronectin but is also present in many other ECM proteins including laminin and collagen (Ruoslahti, 2003). Presentation of RGD on synthetic matrices supports cell adhesion by targeting integrins, such as $\alpha_{v} \beta_{3}$. Many studies have demonstrated successful BM-like bioactivity of RGD-functionalized hydrogels, as compared to nonfunctionalized matrices, by directing essential cellular processes such as attachment and spreading (Schmedlen et al., 2002), migration and invasion (Puperi et al., 2015; Halstenberg et al., 2002), and stem cell support (Nuttelman et al., 2005) and differentiation (Saha et al., 2007). Furthermore, other peptides like IKVAV and YIGSR, which are derived from laminin, can also be incorporated into hydrogels together 
with RGD to orchestrate ligand density-dependent signal presentation independently of the mechanical characteristics of the matrix (Saha et al., 2007; Fittkau et al., 2005). This demonstrates the potential of engineered hydrogels as matrices that can elucidate tunable biochemical signals that direct complex cellular processes via presentation of cell adhesion peptides.

\subsubsection{Protease-Degradable Crosslinkers}

The capacity of cells to modify their microenvironment via matrix degradation is essential for their ability to migrate and proliferate, as well as for tissue remodeling and homeostasis (Lu et al., 2011). ECM remodeling involves degradation and modification of its protein components, most significantly by cell-secreted or membrane-bound matrix metalloproteinases (MMPs) (Sternlicht and Werb, 2001). Therefore, crosslinking hydrogel matrices with MMP-cleavable peptides has been a widely used strategy to allow for cell-directed modifications of the matrix biophysical characteristics. These short peptide sequences are derived from ECM proteins, such as type I or type IV collagen, and incorporation into synthetic hydrogel matrices conveys sensitivity to MMP-dependent degradation (Lutolf et al., 2003; Mann et al., 2001). To incorporate these MMP-cleavable peptides within synthetic hydrogel networks, the peptides are encoded to contain groups that react with the base macromer. For example, a linear MMPcleavable peptide containing $\mathrm{N}$ - and C-terminal cysteines (with free thiols) will react with maleimidefunctionalized PEG macromers via a Michael-type addition reaction (Phelps et al., 2012) (Figure 2c, e). Moreover, studies show that mutations to, or combinations of, different MMP-sensitive crosslinking

peptides can yield hydrogels with different degradability rates that result in variations of cellular responses within the hydrogel, such as extent of cell invasion (Lutolf et al., 2003) and determination of stem cell fate (Das et al., 2015; Rape et al., 2015). Such control over the degradability rate of hydrogels may facilitate the design of synthetic ECM-like materials that present high to low MMP sensitivity that is necessary for short- to long-term matrix stability, respectively. Thus, this demonstrates that artificial hydrogel matrices with tunable degradability rates present BM-like bioactivity that can direct a wide range of cellular responses independent of their cell adhesive peptide density.

\subsubsection{Growth Factor-binding Domains}

The BM hosts a wide variety of signaling molecules such as growth factors (GFs), which reside in the matrix by non-covalent interactions with heparan sulfate proteoglycans such as perlecan and other 
minor BM components (agrin and type XVIII collagen) (Guvendiren and Burdick, 2013; Halfter et al., 1998). GFs play significant roles in tissue development by eliciting a variety of essential cellular responses such as cell proliferation, stem cell differentiation, and vascular and organ morphogenesis (Kleinman et al., 2003). Therefore, presentation of specific GF types and densities to cells through the use of artificial hydrogel matrices has been an effective approach to orchestrate desired cellular responses. The traditional method for GF incorporation into hydrogels is direct encapsulation, which involves physical entrapment of GFs within the matrix which will be released as the hydrogel degrades and/or will diffuse out of the hydrogel network (Sokic and Papavasiliou, 2012). Nevertheless, as this method may not provide long-term control over GF presentation, another approach has been to covalently tether GFs to the hydrogel backbone (Guvendiren and Burdick, 2013; DeLong et al., 2004). However, as covalent linkage may compromise GF activity by alterations to its conformation or masking its active sites, strategies focus on incorporating GF-binding domains into hydrogel matrices by covalently tethering heparan sulfatecontaining molecules to the polymer backbone that interact with GFs (Martino et al., 2013; SakiyamaElbert and Hubbell, 1999; Hudalla and Murphy, 2011). This method, compared to previous approaches, has gained popularity as it mimics native BM-GF non-covalent interactions within artificial hydrogel matrices. Additionally, this strategy facilitates the design of hydrogels that exhibit temporal control over GF sequestration and presentation by functionalization of the polymer backbone with heparan sulfatecontaining molecules that exhibit different affinity levels for different GF types (Martino et al., 2013). Consequently, investigations have demonstrated inherent GF-directed cellular responses via presentation of GF-binding domains within synthetic hydrogels, such as in vivo neovascularization (Jha et al., 2015; Cai et al., 2005) and wound healing (Martino et al., 2013).

\section{Applications of BM-mimicking Hydrogels}

As the fields of cell and matrix biology progress, our understanding of what biological cues are essential for specific cellular processes becomes clearer, and thus, more robust synthetic hydrogels that present these essential biological signals have been engineered. In order to construct such materials, it is critical to understand how changes in the chemical and physical characteristics of hydrogels translate into changes in the local microenvironment of encapsulated cells. The establishment of an artificial hydrogel microenvironment that directs epithelial morphogenesis, controls cell fate, and provides insights into 
cancer progression are among the most recent advances in the field of biomaterials, and various studies are highlighted below.

\subsection{Epithelial Morphogenesis}

Epithelial morphogenesis is part of the organogenesis program of multicellular organisms where the epithelia form transient structures, such as tubules and hollow spherical systems (cysts), that further develop into more complex organs (Martin-Belmonte and Mostov, 2008). During this process, epithelial cells establish tight junctions among neighboring epithelial cells through their lateral membranes, and connections to their supporting BM via integrin receptors in their basal membranes (O'Brien et al., 2002), which contribute to their distinctive cellular polarity (O'Brien et al., 2002; Peng et al., 2014). Studies using 3D collagen and laminin gel cultures have demonstrated that the epithelial morphogenesis developmental program is regulated by biochemical and mechanical signals that result from cell-BM interactions (O'Brien et al., 2002; Yu et al., 2005; Mroue and Bissell, 2013). In these studies, single-cell encapsulation of epithelial cells, such as MDCK cells, results in cell proliferation into a multicellular aggregate and further development of cysts composed of a polarized epithelial monolayer that recreates the morphogenesis of epithelial organs (McAteer et al., 1986). Although these natural matrices have been able to recapitulate the epithelial morphogenesis program of MDCK cells, they do not offer the capacity to study individual contributions of the mechanical and biochemical properties of the ECM towards epithelial morphogenesis. Therefore, Enemchukwu et al (2016) established an engineered ECM-mimetic PEG hydrogel system with independent control over ligand density and presentation, proteolytic degradation and mechanical properties to independently study the effects of ECM biochemical and biophysical properties on MDCK cell morphogenesis. In this study, normal cyst growth, polarization, and lumen formation were restricted to a narrow range of hydrogel elasticity that was controlled by the polymer density. Additionally, RGD density dramatically regulated apicobasal polarity and lumenogenesis independently of cell proliferation, and a threshold level of MMP-directed hydrogel degradation rate was required to regulate these critical epithelial characteristics. This study offers new insights into how ECM biochemical and biophysical properties independently regulate epithelial morphogenetic behaviors, as well as present a platform technology that could potentially be adopted in developmental cell and tumor biology fields to study ECM-directed processes. 


\subsection{D iPSCs Generation}

Since the discovery of induced pluripotent stem cells (iPSCs), reprogramming of somatic cells

has been considered a multi-step process that is initiated by cytoskeletal and epigenetic alterations (Sancho-Martinez and Belmonte, 2013; Polo et al., 2012; Caiazzo et al., 2016). Some of these alterations are related to effects of the ECM on cells, for example, iPSC generation can be influenced by biophysical parameters in 2D culture (Downing et al., 2013). In order to better understand the role of the microenvironment on somatic-cell reprogramming, a synthetic 3D ECM-like hydrogel culture system that supports the initiation of reprogramming was developed by Lutolf (Caiazzo et al., 2016). These PEG hydrogels allow for precise control of the physiochemical characteristics of the cellular microenvironment which is not possible in 2D culture. In order to take full advantage of the tunable nature of this hydrogel, a high-throughput imaging system was used to detect pluripotency levels as a function of hydrogel stiffness, susceptibility to MMP degradation, and functionalization with different proteins previously shown to play a role in regulating pluripotency. This analysis yielded an engineered 3D PEG hydrogel that demonstrated a 2.5-fold higher reprogramming efficiency of human fibroblasts into iPSCs as compared to $2 \mathrm{D}$ culture. The accelerated reprogramming was attributed to hydrogel matrix selection of colony-forming iPSCs by limiting proliferation of non-colony-forming cells, and to the pronounced morphological changes that may cause the key events for the initiation of iPSC generation. These findings provide the first proof of principle for $3 \mathrm{D}$ reprogramming in synthetic matrices, and demonstrate the capacity of an engineered ECM-like hydrogel system to reveal the influence of the physiochemical characteristics of the cellular microenvironment in cell fate regulation.

\subsection{Tumor Progression}

Genetic changes in tumor cells and the biochemical and biophysical properties of the local microenvironment have been major attributions to cancer progression and metastasis (Hanahan and Weinberg, 2011; Bissell and Radisky, 2001; Provenzano et al., 2009). The effect of changes in the local microenvironment have been evaluated through 2D culture models and, although they have been contributory in the understanding of tumor progression, they do not recapitulate the complex properties of the 3D in vivo microenvironment (Bissell and Radisky, 2001; Nelson and Bissell, 2005). Consequently, Weaver, Bissell and others have done seminal work in studying how abnormalities in the ECM contribute 
to tumor progression through the use of 3D collagen and laminin (i.e., Matrigel ${ }^{\mathrm{TM}}$ ) gels (Levental et al., 2009; Egeblad et al., 2010; Weaver et al., 1997; Lu et al., 2012). Whereas these naturally derived materials can recapitulate complex features of native ECM, results are often inconsistent and/or difficult to interpret due to batch-to-batch variability and undefined composition (Hughes et al., 2010). Therefore, in order to further comprehend the effects of the local microenvironment in tumor progression in a 3D culture while overcoming the principal limitations of naturally derived materials, Singh et al. (2015) developed an engineered PEG-based synthetic hydrogel system that presents a cell adhesive peptide motif (RGD) and MMP-degradable crosslinks that induce cell adhesion and cell-directed degradability. In this study, this ECM-like synthetic hydrogel served as a cellular niche that promoted the formation of human melanoma cell (WM239A) clusters from a single cell in a matrix stiffness-dependent manner. Furthermore, to study cancer cell migration and invasion, melanoma cell migration within the hydrogel system was studied in the presence of human dermal fibroblasts, which resulted in cell migration to surrounding regions in a way that resembled invasion of patient-derived melanoma xenograft tumors ( $\mathrm{Li}$ et al., 2003; Hu et al., 2008). Taken together, these results showed the application of a synthetic 3D hydrogel culture system that models the growth, migration and invasion of a human melanoma cell line or xenograft tumors, and at the same time serves as a tool to study the role for several critical components of the microenvironment during tumor progression.

\section{Outlook for BM-like Synthetic Hydrogel Matrices}

Although the studies discussed in this review have developed novel artificial hydrogel matrices that present BM-like properties, several challenges need to be addressed in order to continue engineering synthetic matrices that recapitulate and direct complex cellular processes. For instance, there is a need for hydrogels that can recapitulate the in vivo microenvironment by presentation of multiple adhesive ligand types at specific densities that can orchestrate complex cellular responses. Such artificial matrix designs are important for the support of complex cellular systems (e.g. primary and stem cells) that still represent a challenge due to their increased sensitivity, and to further direct innate cellular functions. Furthermore, as different studies have demonstrated successful culture of complex cellular systems using Matrigel ${ }^{\mathrm{TM}}$, future developments of BM-like synthetic hydrogels should move towards designing hydrogels that recapitulate mechanical and structural (e.g., fibrillar structure) properties of Matrigel ${ }^{\mathrm{TM}}$ and collagen gels. 
Consequently, designing artificial matrices that present combinations of biochemical signals by functionalization with multiple adhesive ligand types, and possess biophysical characteristics of Matrigel $^{\mathrm{TM}}$, may lead to hydrogels that reiterate the in vivo microenvironment of complex cellular systems and can, thus, further expand their application as cell-delivery vehicles for in vivo studies. Therefore, as new ways of integrating bioactivity into hydrogel matrices are designed, these novel engineered synthetic materials will continue to offer new developments in regenerative medicine and tissue engineering fields, and promise innovative therapeutic options that naturally derived materials cannot provide.

Acknowledgements. This work was funded by National Institutes of Health (NIH) grants R01AR062368 and R01-AR062920 and a seed grant from the Regenerative Engineering and Medicine Research Center between Emory University, Georgia Tech and The University of Georgia. RCA is supported by a National Science Foundation Graduate Research Fellowship.

\section{References:}

1. Anseth, Kristi S., Christopher N. Bowman, and Lisa Brannon-Peppas. "Mechanical Properties of Hydrogels and Their Experimental Determination." Biomaterials 17.17 (1996): 1647-57. Print.

2. Barcellos-Hoff, M. H., et al. "Functional Differentiation and Alveolar Morphogenesis of Primary Mammary Cultures on Reconstituted Basement Membrane." Development (Cambridge, England) 105.2 (1989): 223-35. Print.

3. Beck, J. N., et al. "The Independent Roles of Mechanical, Structural and Adhesion Characteristics of 3d Hydrogels on the Regulation of Cancer Invasion and Dissemination." Biomaterials 34.37 (2013): 9486-95. Print.

4. Cai, S., et al. "Injectable Glycosaminoglycan Hydrogels for Controlled Release of Human Basic Fibroblast Growth Factor." Biomaterials 26.30 (2005): 6054-67. Print.

5. Caiazzo, M., et al. "Defined Three-Dimensional Microenvironments Boost Induction of Pluripotency." Nat Mater 15.3 (2016): 344-52. Print.

6. $\quad$ Chaudhuri, O., et al. "Hydrogels with Tunable Stress Relaxation Regulate Stem Cell Fate and Activity." Nat Mater 15.3 (2016): 326-34. Print.

7. Cheng, Yi-Shan, et al. "Self-Assembly of Laminin Isoforms." Journal of Biological Chemistry 272.50 (1997): 31525-32. Print. 
8. Colognato, Holly, and Peter D. Yurchenco. "Form and Function: The Laminin Family of Heterotrimers." Developmental Dynamics 218.2 (2000): 213-34. Print.

9. Danen, E. H., and A. Sonnenberg. "Integrins in Regulation of Tissue Development and Function." J Pathol 201.4 (2003): 632-41. Print.

10. Danen, Erik H. J. "Integrins: Regulators of Tissue Function and Cancer Progression." Current Pharmaceutical Design 11.7 (2005): 881-91. Print.

11. Das, R. K., et al. "Stress-Stiffening-Mediated Stem-Cell Commitment Switch in Soft Responsive Hydrogels." Nat Mater 15.3 (2016): 318-25. Print.

12. Debnath, Jayanta, Senthil K. Muthuswamy, and Joan S. Brugge. "Morphogenesis and Oncogenesis of Mcf-10a Mammary Epithelial Acini Grown in Three-Dimensional Basement Membrane Cultures." Methods 30.3 (2003): 256-68. Print.

13. DeLong, S. A., J. J. Moon, and J. L. West. "Covalently Immobilized Gradients of Bfgf on Hydrogel Scaffolds for Directed Cell Migration." Biomaterials 26.16 (2005): 3227-34. Print.

14. Downing, T. L., et al. "Biophysical Regulation of Epigenetic State and Cell Reprogramming." Nat Mater 12.12 (2013): 1154-62. Print.

15. Drury, Jeanie L., and David J. Mooney. "Hydrogels for Tissue Engineering: Scaffold Design Variables and Applications." Biomaterials 24.24 (2003): 4337-51. Print.

16. Durbeej, Madeleine, et al. "Expression of Laminin A1, A5 and B2 Chains During Embryogenesis of the Kidney and Vasculature." Matrix Biology 15.6 (1996): 397-413. Print.

17. Egeblad, M., M. G. Rasch, and V. M. Weaver. "Dynamic Interplay between the Collagen Scaffold and Tumor Evolution." Curr Opin Cell Biol 22.5 (2010): 697-706. Print.

18. Ekblom, Marja, et al. "Laminin Isoforms and Epithelial Development." Annals of the New York Academy of Sciences 857.1 (1998): 194-211. Print.

19. Enemchukwu, N. O., et al. "Synthetic Matrices Reveal Contributions of Ecm Biophysical and Biochemical Properties to Epithelial Morphogenesis." J Cell Biol 212.1 (2016): 113-24. Print.

20. Engbring, J. A., and H. K. Kleinman. "The Basement Membrane Matrix in Malignancy." J Pathol 200.4 (2003): 465-70. Print.

21. Fatehullah, A., S. H. Tan, and N. Barker. "Organoids as an in Vitro Model of Human Development and Disease." Nat Cell Biol 18.3 (2016): 246-54. Print.

22. Fittkau, M. H., et al. "The Selective Modulation of Endothelial Cell Mobility on Rgd Peptide Containing Surfaces by Yigsr Peptides." Biomaterials 26.2 (2005): 167-74. Print.

23. Flory, Paul J., and John Rehner. "Statistical Mechanics of Cross-Linked Polymer Networks Ii. Swelling." The Journal of Chemical Physics 11.11 (1943): 521. Print. 
24. Gaggioli, C., et al. "Fibroblast-Led Collective Invasion of Carcinoma Cells with Differing Roles for Rhogtpases in Leading and Following Cells." Nat Cell Biol 9.12 (2007): 1392-400. Print.

25. Gallant, N. D., and A. J. Garcia. "Model of Integrin-Mediated Cell Adhesion Strengthening." J Biomech 40.6 (2007): 1301-9. Print.

26. Garcia, A. J. "Get a Grip: Integrins in Cell-Biomaterial Interactions." Biomaterials 26.36 (2005): 7525-9. Print.

27. Garcia, A. J. "Peg-Maleimide Hydrogels for Protein and Cell Delivery in Regenerative Medicine." Ann Biomed Eng 42.2 (2014): 312-22. Print.

28. Garcia, J. R., and A. J. Garcia. "Biomaterial-Mediated Strategies Targeting Vascularization for Bone Repair." Drug Deliv Transl Res 6.2 (2016): 77-95. Print.

29. Giancotti, Filippo G, and Erkki Ruoslahti. "Integrin Signaling." Science 285.5430 (1999): 1028-33. Print.

30. Guvendiren, M., and J. A. Burdick. "Engineering Synthetic Hydrogel Microenvironments to Instruct Stem Cells." Curr Opin Biotechnol 24.5 (2013): 841-6. Print.

31. Halfter, Willi, et al. "Collagen Xviii Is a Basement Membrane Heparan Sulfate Proteoglycan." Journal of Biological Chemistry 273.39 (1998): 25404-12. Print.

32. Halstenberg, Sven, et al. "Biologically Engineered Protein-Graft-Poly(Ethylene Glycol) Hydrogels: A Cell Adhesive and Plasmin-Degradable Biosynthetic Material for Tissue Repair." Biomacromolecules 3.4 (2002): 710-23. Print.

33. Hanahan, D., and R. A. Weinberg. "Hallmarks of Cancer: The Next Generation." Cell 144.5 (2011): 646-74. Print.

34. Hoffman, Allan S. "Hydrogel for Biomedical Applications." Advanced Drug Delivery Reviews 54.1 (2002): 3-12. Print.

35. Hu, BH., Jing Su, and P.B. Messersmith. "Hydrogels Cross-Linked by Native Chemical Ligation." Biomacromolecules 10.8 (2009): 2194-200. Print.

36. Hu, Min. "Regulation of in Situ to Invasive Breast Carcinoma Transition." 2008. Print.

37. Hudalla, G. A., and W. L. Murphy. "Biomaterials That Regulate Growth Factor Activity Via Bioinspired Interactions." Adv Funct Mater 21.10 (2011): 1754-68. Print.

38. Hughes, C. S., L. M. Postovit, and G. A. Lajoie. "Matrigel: A Complex Protein Mixture Required for Optimal Growth of Cell Culture." Proteomics 10.9 (2010): 1886-90. Print.

39. Hutson, C. B., et al. "Synthesis and Characterization of Tunable Poly(Ethylene Glycol): Gelatin Methacrylate Composite Hydrogels." Tissue Eng Part A 17.13-14 (2011): 1713-23. Print.

40. Huttenlocher, A., and A. R. Horwitz. "Integrins in Cell Migration." Cold Spring Harb Perspect Biol 3.9 (2011): a005074. Print.

41. Hynes, R. O. "The Extracellular Matrix: Not Just Pretty Fibrils." Science 326.5957 (2009): 1216-9. Print.

42. Hynes, Richard O. "Integrins: Bidirectional, Allosteric Signaling Machines." Cell 110.6 (2002): 673-87. Print.

43. Hynes, Richard O. "Stretching the Boundaries of Extracellular Matrix Research." Nature Reviews Molecular Cell Biology 15.12 (2014): 761-63. Print. 
44. Janmey, P. A., J. P. Winer, and J. W. Weisel. "Fibrin Gels and Their Clinical and Bioengineering Applications." J R Soc Interface 6.30 (2009): 1-10. Print.

45. Jha, A. K., et al. "Enhanced Survival and Engraftment of Transplanted Stem Cells Using Growth Factor Sequestering Hydrogels." Biomaterials 47 (2015): 1-12. Print.

46. Kalluri, R. "Basement Membranes: Structure, Assembly and Role in Tumour Angiogenesis." Nat Rev Cancer 3.6 (2003): 422-33. Print.

47. Khetan, S., et al. "Degradation-Mediated Cellular Traction Directs Stem Cell Fate in Covalently Crosslinked ThreeDimensional Hydrogels." Nat Mater 12.5 (2013): 458-65. Print.

48. Kikkawa, Y., et al. "Laminin-111-Derived Peptides and Cancer." Cell Adh Migr 7.1 (2013): 150-256. Print.

49. Kim, I. L., R. L. Mauck, and J. A. Burdick. "Hydrogel Design for Cartilage Tissue Engineering: A Case Study with Hyaluronic Acid." Biomaterials 32.34 (2011): 8771-82. Print.

50. Kleinman, Hynda K., Deborah Philp, and Matthew P. Hoffman. "Role of the Extracellular Matrix in Morphogenesis." Current Opinion in Biotechnology 14.5 (2003): 526-32. Print.

51. Kloxin, April M., et al. "Photodegradable Hydrogels for Dynamic Tuning of Physical and Chemical Properties." Science 324.5923 (2009): 59-63. Print.

52. Kloxin, A. M., et al. "Mechanical Properties of Cellularly Responsive Hydrogels and Their Experimental Determination." Adv Mater 22.31 (2010): 3484-94. Print.

53. Kloxin, A. M., M. W. Tibbitt, and K. S. Anseth. "Synthesis of Photodegradable Hydrogels as Dynamically Tunable Cell Culture Platforms." Nat Protoc 5.12 (2010): 1867-87. Print.

54. Lam, J., N. F. Truong, and T. Segura. "Design of Cell-Matrix Interactions in Hyaluronic Acid Hydrogel Scaffolds." Acta Biomater 10.4 (2014): 1571-80. Print.

55. LeBleu, V.S., B. MacDonald, and R. Kalluri. "Structure and Function of Basement Membranes." Exp Biol Med 232.9 (2007): 1121-29. Print.

56. Lee, K. Y., and D. J. Mooney. "Alginate: Properties and Biomedical Applications." Prog Polym Sci 37.1 (2012): 106-26. Print.

57. Lee, T. T., et al. "Light-Triggered in Vivo Activation of Adhesive Peptides Regulates Cell Adhesion, Inflammation and Vascularization of Biomaterials." Nat Mater 14.3 (2015): 352-60. Print.

58. Levental, K. R., et al. "Matrix Crosslinking Forces Tumor Progression by Enhancing Integrin Signaling." Cell 139.5 (2009): 891-906. Print.

59. Li, G., et al. "Function and Regulation of Melanoma-Stromal Fibroblast Interactions: When Seeds Meet Soil." Oncogene 22.20 (2003): 3162-71. Print.

60. Li, J., et al. "Integrin Beta4 Regulation of Pthrp Underlies Its Contribution to Mammary Gland Development." Dev Biol 407.2 (2015): 313-20. Print. 
61. Lu, P., et al. "Extracellular Matrix Degradation and Remodeling in Development and Disease." Cold Spring Harb Perspect Biol 3.12 (2011). Print.

62. Lu, P., V. M. Weaver, and Z. Werb. "The Extracellular Matrix: A Dynamic Niche in Cancer Progression." J Cell Biol 196.4 (2012): 395-406. Print.

63. Lukashev, Matvey E, and Zena Werb. "Ecm Signalling: Orchestrating Cell Behaviour and Misbehaviour." Trends in cell biology 8.11 (1998): 437-41. Print.

64. Lutolf, M. P., et al. "Synthetic Matrix Metalloproteinase-Sensitive Hydrogels for the Conduction of Tissue Regeneration: Engineering Cell-Invasion Characteristics." Proc Natl Acad Sci U S A 100.9 (2003): 5413-8. Print.

65. Mann, Brenda K, et al. "Smooth Muscle Cell Growth in Photopolymerized Hydrogels with Cell Adhesive and Proteolytically Degradable Domains: Synthetic Ecm Analogs for Tissue Engineering." Biomaterials 22.22 (2001): 3045-51. Print.

66. Martin-Belmonte, F., and K. Mostov. "Regulation of Cell Polarity During Epithelial Morphogenesis." Curr Opin Cell Biol 20.2 (2008): 227-34. Print.

67. Martino, M. M., et al. "Heparin-Binding Domain of Fibrin(Ogen) Binds Growth Factors and Promotes Tissue Repair When Incorporated within a Synthetic Matrix." Proc Natl Acad Sci U S A 110.12 (2013): 4563-8. Print.

68. Martins-Green, M., and M. J. Bissell. "Cell-Ecm Interactions in Development." Seminars in Developmental Biology 6.2 (1995): 149-59. Print.

69. Matricardi, P., et al. "Interpenetrating Polymer Networks Polysaccharide Hydrogels for Drug Delivery and Tissue Engineering." Adv Drug Deliv Rev 65.9 (2013): 1172-87. Print.

70. McAteer, James A., et al. "Mdck Cysts: An in Vitro Model of Epithelial Cyst Formation and Growth." Journal of tissue culture methods 10.4: 245-48. Print.

71. Midwood, K. S., L. V. Williams, and J. E. Schwarzbauer. "Tissue Repair and the Dynamics of the Extracellular Matrix." Int J Biochem Cell Biol 36.6 (2004): 1031-7. Print.

72. Montesano, R, G Schaller, and L Orci. "Induction of Epithelial Tubular Morphogenesis in Vitro by Fibroblast-Derived Soluble Factors." Cell 66.4 (1991): 697-711. Print.

73. Mroue, R., and M. J. Bissell. "Three-Dimensional Cultures of Mouse Mammary Epithelial Cells." Methods Mol Biol 945 (2013): 221-50. Print.

74. Nelson, C. M., and M. J. Bissell. "Modeling Dynamic Reciprocity: Engineering Three-Dimensional Culture Models of Breast Architecture, Function, and Neoplastic Transformation." Semin Cancer Biol 15.5 (2005): 342-52. Print.

75. Nguyen, Kytai Truong, and Jennifer L. West. "Photopolymerizable Hydrogels for Tissue Engineering Applications." Biomaterials 23.22 (2002): 4307-14. Print.

76. Nguyen-Ngoc, K. V., et al. "Ecm Microenvironment Regulates Collective Migration and Local Dissemination in Normal and Malignant Mammary Epithelium." Proc Natl Acad Sci U S A 109.39 (2012): E2595-604. Print. 
77. Nuttelman, C. R., M. C. Tripodi, and K. S. Anseth. "Synthetic Hydrogel Niches That Promote Hmsc Viability." Matrix Biol 24.3 (2005): 208-18. Print.

78. O'Brien, Lucy Erin, et al. "Rac1 Orientates Epithelial Apical Polarity through Effects on Basolateral Laminin Assembly." Nature cell biology 3.9 (2001): 831-38. Print.

79. O'Brien, Lucy Erin, Mirjam M. P. Zegers, and Keith E. Mostov. "Opinion: Building Epithelial Architecture: Insights from Three-Dimensional Culture Models." Nature Reviews. Molecular Cell Biology 3.7 (2002): 531-37. Print.

80. Pati, F., et al. "Ornamenting 3d Printed Scaffolds with Cell-Laid Extracellular Matrix for Bone Tissue Regeneration." Biomaterials 37 (2015): 230-41. Print.

81. Peng, J., et al. "Phosphoinositide 3-Kinase P110delta Promotes Lumen Formation through the Enhancement of ApicoBasal Polarity and Basal Membrane Organization." Nat Commun 6 (2015): 5937. Print.

82. Peppas, N. A., et al. "Physicochemical Foundations and Structural Design of Hydrogels in Medicine and Biology." Annual Review of Biomedical Engineering 2.1 (2000): 9-29. Print.

83. Phelps, E. A., et al. "Maleimide Cross-Linked Bioactive Peg Hydrogel Exhibits Improved Reaction Kinetics and CrossLinking for Cell Encapsulation and in Situ Delivery." Adv Mater 24.1 (2012): 64-70, 2. Print.

84. Polizzotti, Brian D., Benjiman D. Fairbanks, and Kristi S. Anseth. "Three-Dimensional Biochemical Patterning of Click-Based Composite Hydrogels Via Thiolene Photopolymerization." Biomacromolecules 9.4 (2008): 1084-87. Print.

85. Polo, J. M., et al. "A Molecular Roadmap of Reprogramming Somatic Cells into Ips Cells." Cell 151.7 (2012): 1617-32. Print.

86. Provenzano, P. P., et al. "Matrix Density-Induced Mechanoregulation of Breast Cell Phenotype, Signaling and Gene Expression through a Fak-Erk Linkage." Oncogene 28.49 (2009): 4326-43. Print.

87. Puperi, D. S., et al. "3-Dimensional Spatially Organized Peg-Based Hydrogels for an Aortic Valve Co-Culture Model." Biomaterials 67 (2015): 354-64. Print.

88. Rape, A. D., et al. "A Synthetic Hydrogel for the High-Throughput Study of Cell-Ecm Interactions." Nat Commun 6 (2015): 8129. Print.

89. Riopel, M., M. Trinder, and R. Wang. "Fibrin, a Scaffold Material for Islet Transplantation and Pancreatic Endocrine Tissue Engineering." Tissue Eng Part B Rev 21.1 (2015): 34-44. Print.

90. Ruoslahti, Erkki. "The Rgd Story: A Personal Account." Matrix Biology 22.6 (2003): 459-65. Print.

91. Saha, K., et al. "Biomimetic Interfacial Interpenetrating Polymer Networks Control Neural Stem Cell Behavior." J Biomed Mater Res A 81.1 (2007): 240-9. Print.

92. Sakiyama-Elbert, Shelly E, and Jeffrey A Hubbell. "Development of Fibrin Derivatives for Controlled Release of Heparin-Binding Growth Factors." Journal of Controlled Release 65.3 (2000): 389-402. Print.

93. Sancho-Martinez, Ignacio, and Juan Carlos Izpisua Belmonte. "Stem Cells: Surf the Waves of Reprogramming." Nature 493.7432 (2013): 310-11. Print. 
94. Sato, T., and H. Clevers. "Growing Self-Organizing Mini-Guts from a Single Intestinal Stem Cell: Mechanism and Applications." Science 340.6137 (2013): 1190-4. Print.

95. Sato, T., et al. "Single Lgr5 Stem Cells Build Crypt-Villus Structures in Vitro without a Mesenchymal Niche." Nature 459.7244 (2009): 262-5. Print.

96. Schmedlen, Rachael H, Kristyn S Masters, and Jennifer L West. "Photocrosslinkable Polyvinyl Alcohol Hydrogels That Can Be Modified with Cell Adhesion Peptides for Use in Tissue Engineering." Biomaterials 23.22 (2002): 4325-32. Print.

97. Schwartz, Michael P., et al. "Human Pluripotent Stem Cell-Derived Neural Constructs for Predicting Neural Toxicity." Proceedings of the National Academy of Sciences 112.40 (2015): 12516-21. Print.

98. Shamir, E. R., and A. J. Ewald. "Three-Dimensional Organotypic Culture: Experimental Models of Mammalian Biology and Disease." Nat Rev Mol Cell Biol 15.10 (2014): 647-64. Print.

99. Siler, Ulrich, et al. "Characterization and Functional Analysis of Laminin Isoforms in Human Bone Marrow." Blood 96.13 (2000): 4194-203. Print.

100. Singh, S. P., et al. "A Synthetic Modular Approach for Modeling the Role of the 3d Microenvironment in Tumor Progression." Sci Rep 5 (2015): 17814. Print.

101. Sokic, S., and G. Papavasiliou. "Fgf-1 and Proteolytically Mediated Cleavage Site Presentation Influence ThreeDimensional Fibroblast Invasion in Biomimetic Pegda Hydrogels." Acta Biomater 8.6 (2012): 2213-22. Print.

102. Sternlicht, Mark D, and Zena Werb. "How Matrix Metalloproteinases Regulate Cell Behavior." Annual review of cell and developmental biology 17 (2001): 463. Print.

103. Streuli, Charles. "Extracellular Matrix Remodelling and Cellular Differentiation." Current opinion in cell biology 11.5 (1999): 634-40. Print.

104. Thiele, J., et al. "25th Anniversary Article: Designer Hydrogels for Cell Cultures: A Materials Selection Guide." Adv Mater 26.1 (2014): 125-47. Print.

105. Turpeenniemi-Hujanen, T, et al. "Laminin Increases the Release of Type IV Collagenase from Malignant Cells." Journal of Biological Chemistry 261.4 (1986): 1883-89. Print.

106. Weaver, Valerie M, et al. "Reversion of the Malignant Phenotype of Human Breast Cells in Three-Dimensional Culture and in Vivo by Integrin Blocking Antibodies." The Journal of cell biology 137.1 (1997): 231-45. Print.

107. Wolf, K., et al. "Collagen-Based Cell Migration Models in Vitro and in Vivo." Semin Cell Dev Biol 20.8 (2009): 931-41. Print.

108. Yu, Wei, et al. "B1-Integrin Orients Epithelial Polarity Via Rac1 and Laminin." Molecular Biology of the Cell 16.2 (2005): 433-45. Print.

109. Yue, K., et al. "Synthesis, Properties, and Biomedical Applications of Gelatin Methacryloyl (Gelma) Hydrogels." Biomaterials 73 (2015): 254-71. Print. 
110. Yui, S., et al. "Functional Engraftment of Colon Epithelium Expanded in Vitro from a Single Adult Lgr5(+) Stem Cell." Nat Med 18.4 (2012): 618-23. Print.

111. Yurchenco, P. D. "Basement Membranes: Cell Scaffoldings and Signaling Platforms." Cold Spring Harb Perspect Biol 3.2 (2011). Print.

112. Zhang, L., E. M. Furst, and K. L. Kiick. "Manipulation of Hydrogel Assembly and Growth Factor Delivery Via the Use of Peptide-Polysaccharide Interactions." J Control Release 114.2 (2006): 130-42. Print.

113. Zhu, J. "Bioactive Modification of Poly(Ethylene Glycol) Hydrogels for Tissue Engineering." Biomaterials 31.17 (2010): 4639-56. Print.

Figure Legends:

Figure 1: Three-dimensional arrangement of basement membrane components and its interaction with cellular receptors. Based on LeBleu et al., 2007 (A) BM-directed cellular response is primarily 
initiated by interactions between integrin cell receptors and specific peptide motifs in BM proteins. (B) Three-dimensional arrangement of the four major basement membrane components.

Figure 2: Synthesis methods of engineered synthetic hydrogels. Photo-polymerization of two watersoluble polymers (A) and copolymerization of one monomer and one multi-functional monomer (B) via covalent reaction after UV irradiation. (C) Direct chemical reaction of a branched polymer with a difunctional crosslinking agent. (D) Electrostatic interactions of an anionic polymer with divalent cations yields an "ionotropic" hydrogel. (E) Encapsulated cells in an engineered biofunctionalized hydrogel exhibiting adhesive ligand, growth factor-binding domain and MMP-degradable crosslinks.

Figure 1: 


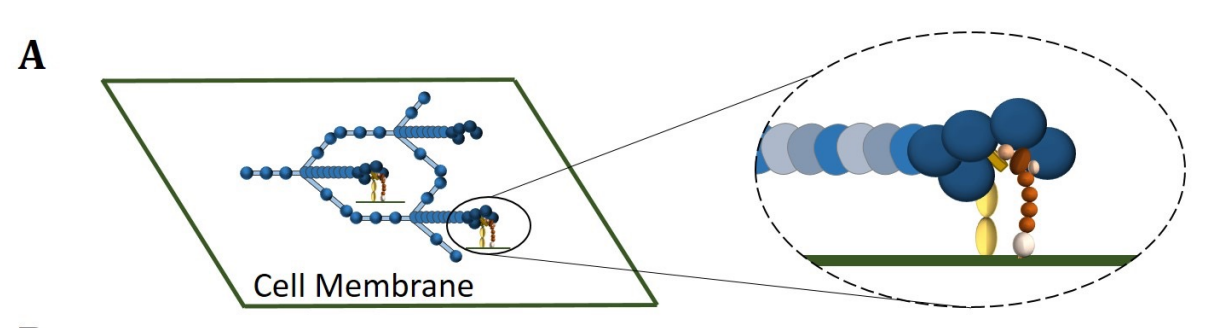

B

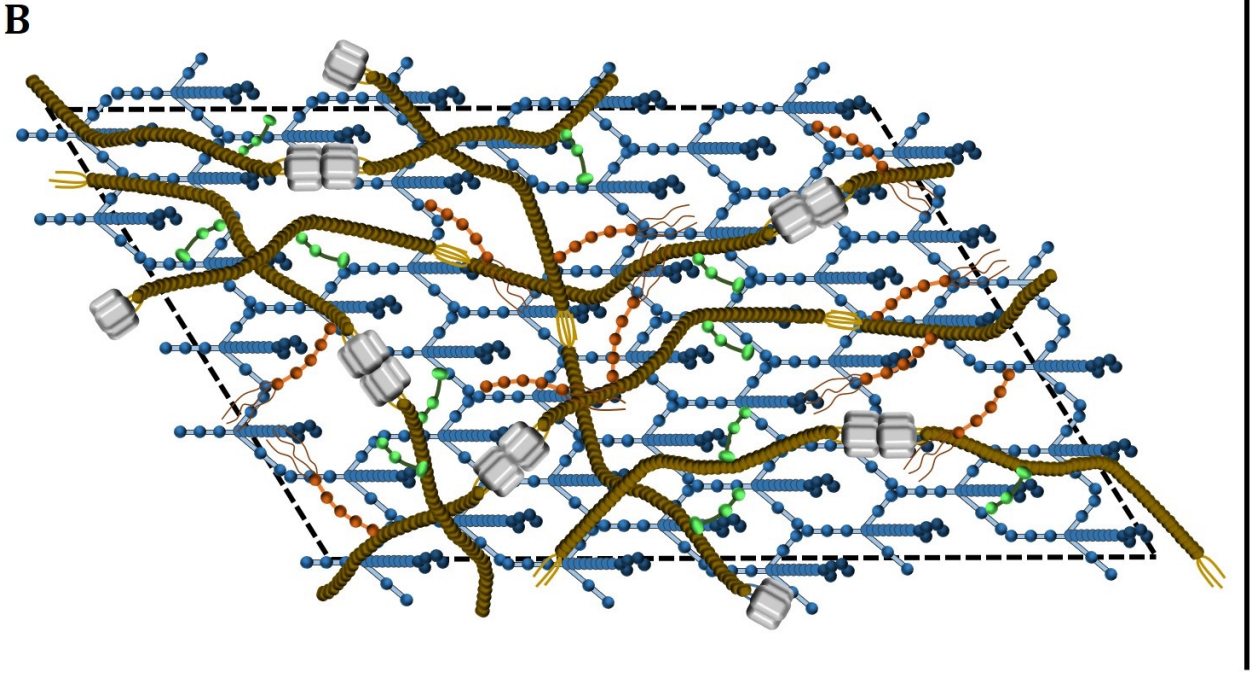

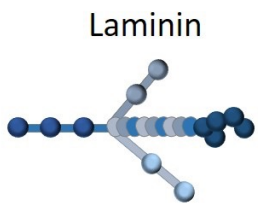

Integrin

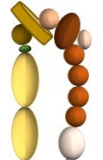

Perlecan

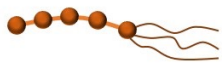

Nidogen

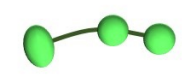

Type IV Collagen

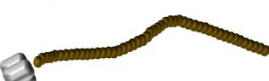

Figure 2: 
A

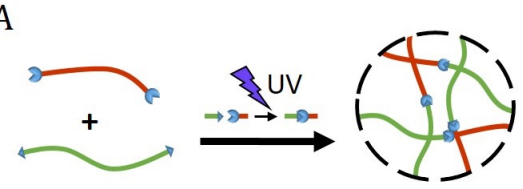

B

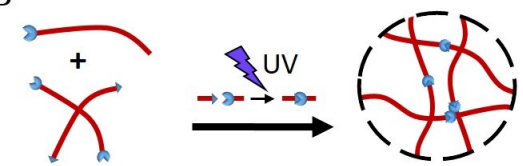

C

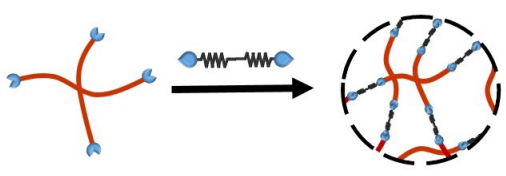

D

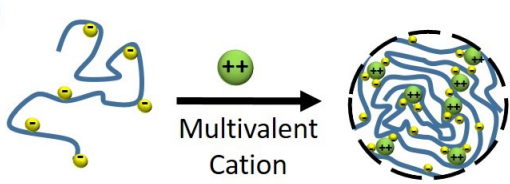

E

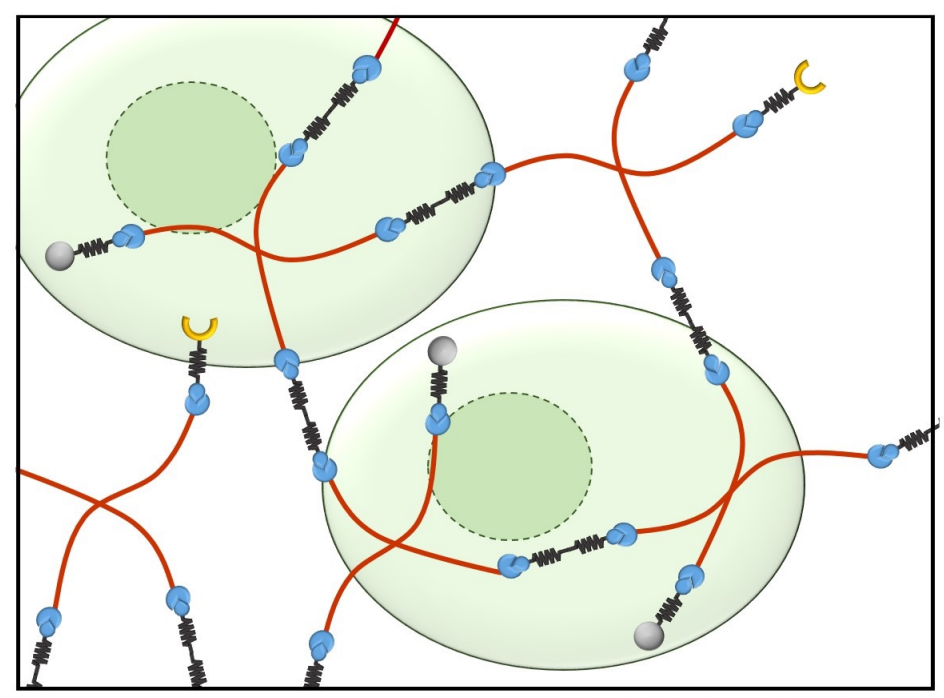

Crosslinking Adhesive Growth Factor- Polymer Backbone Peptide

oun-whe Cure S-unro
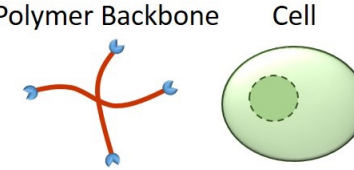